\title{
Influence of the Aperture-Based Receiver Orientation on RSS-Based VLP Performance.
}

\author{
José Miguel Menéndez ${ }^{1,2}$, Heidi Steendam ${ }^{1}$ \\ ${ }^{1}$ Dept. of Telecommunications and Information Processing, Ghent University, Ghent, Belgium, \\ Email: \{JoseMiguel.MenendezSanchez,Heidi.Steendam\}@ugent.be \\ ${ }^{2}$ Dept. of Electrical and Computer Engineering, ESPOL University, Campus Gustavo Galindo, Guayaquil, Ecuador \\ Email: jmmnend@espol.edu.ec
}

\begin{abstract}
Recently, a compact receiver structure, containing a set of apertures and photo diodes (PDs) that are arranged to offer good angular diversity, was proposed for visible light communication [1]. In [2], this receiver structure was considered for visual light positioning (VLP), where the positioning accuracy for direct estimation of the position was evaluated using the Cramer-Rao Lower bound. However, it was assumed that the receiver is parallel to the ceiling, an assumption that is not realistic in practice. Therefore, in this paper, we extend the results of [2] to include the orientation of the receiver in the performance evaluation. The results show that, provided that the receiver knows its orientation, a rotation of the receiver has essentially no effect on the performance. Further, as long as sufficient LEDs are within the field-of-view of the receiver, the performance degradation will be small. This will be the case for a wide range of tilt angles. Hence, we can conclude that centimetre accuracy can be obtained for a wide range of receiver orientations.
\end{abstract}

Index Terms-VLP, Cramer-Rao Lower bound

\section{INTRODUCTION}

Positioning in indoor environments received a lot of attention in the research community during the last decade. Several approaches were investigated, where most approaches are based on the reception of radio frequency (RF) signals, e.g. WiFi, Bluetooth or Ultra Wideband (UWB) [3], [4], [5]. Recently, Visible Light Positioning (VLP) was considered [6]. In this approach, Light Emitting Diodes (LEDs), that are available for illumination purposes, are modulated to also serve as anchors for a positioning network. Besides the advantage that the positioning network can coexist with illumination, which reduces the installation and maintenance cost, the VLP system also benefits from other advantages such as its low power consumption and the lifetime [7], [8], [9]. Moreover, the position accuracy that is reported in the literature, i.e. an accuracy of the order of centimetres, is among the best of all indoor positioning approaches [10].

Most recent approaches consider a receiver containing one or more photo diodes (PD) to extract position related features from the emitted light, e.g. the received signal strength (RSS) [11], [12], [13], the angle-of-arrival (AOA) [14], [15] or the time-of-arrival (TOA) [16], [17]. Among these techniques, we distinguish the approaches that make use of the knowledge of the radiation pattern of the LEDs, i.e. the optical power emitted by a LED in general follows a Lambertian pattern, where the optical power radiated in a given direction is a function of the angle of that direction to the normal of the LED. For these techniques, the receiver can either directly estimate its position from the detected light [2], [12], or it can first determine the distance to or angle with each LED, and then estimate its position using geometrical techniques [11], [14], [18]. Although the latter has lower complexity, the former has better positioning accuracy.

In this paper, we consider the direct estimation of the position using an aperture-based receiver [1]. In [2], the Cramer-Rao Bound (CRB) on the position, which is a lower bound on the mean-squared error (MSE) of the position, was evaluated, and centimetre accuracy was reported. However, in [2] it is assumed that the receiver is parallel to the ceiling, which is not realistic in practice. In this paper, we therefore extend the work from [2] in order to include the receiver orientation in the CRB, assuming this orientation is known. This assumption is realistic as the orientation of a device can be estimated accurately with an Inertial Measurement Unit (IMU), which nowadays is included in most mobile devices. Our results show that the positioning performance is largely insensitive to the receiver orientation: for a wide range of tilt angles and rotation angles, the performance degradation is negligible.

\section{SYSTEM DESCRIPTION}

\section{A. Received Signals}

The considered visible light positioning system consists of $K$ transmitters, i.e. the white LEDs, and a receiver. The position of the receiver is determined based on the incoming light broadcast by the different LEDs, and the knowledge of the positions of the LEDs. To determine the position of the receiver, we define the coordinate system $X Y Z$ that is fixed to the room and describe the positions of the LEDs and the receiver in the room. The LEDs, which are attached on the ceiling and point downwards, have coordinates $\boldsymbol{v}_{S, i}=\left(x_{S, i}, y_{S, i}, z_{S, i}\right)^{T}$, and are modelled as generalized Lambertian LEDs with order $m_{i}$, $i=1, \ldots, K$. Further, on the receiver, we select a reference point of which the coordinates $\boldsymbol{v}_{U}=\left(x_{U}, y_{U}, z_{U}\right)^{T}$ must be determined in the positioning process. The receiver, which is shown in Fig. 1a, contains $M$ receiving elements (REs), each consisting of a bare photo diode (PD) and an aperture. The PDs and apertures are arranged in parallel planes, where the plane of the apertures is at a height $h_{A}$ above the plane of 


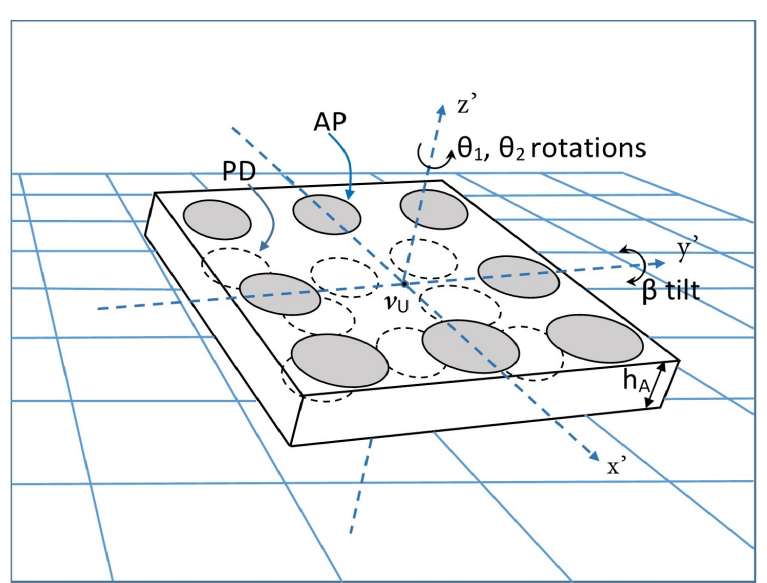

(a)

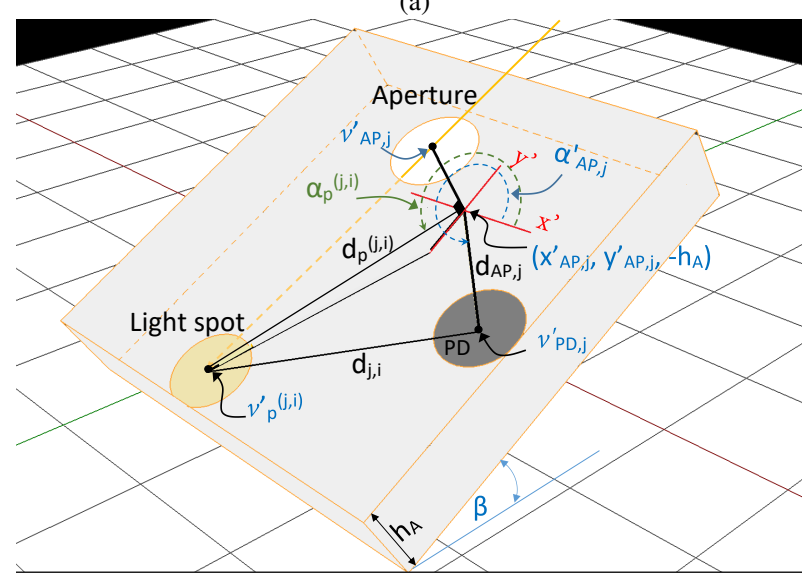

(b)

Fig. 1: Rotation axes for $\theta_{1}, \beta$ and $\theta_{2}$, a) Top view of the receiver, b) A 3D representation of the receiver.

the PDs. The reference point $\boldsymbol{v}_{U}$ of the receiver is selected in the plane of the apertures. The receiver is in general not parallel to the ceiling of the room. Hence, we first introduce the coordinate system $X^{\prime} Y^{\prime} Z^{\prime}$ that is attached to the receiver and has as origin the reference point $\boldsymbol{v}_{U}$ as shown in Fig. 1a. The orientation of the receiver is determined by the Euler angles $\theta_{1}, \beta$ and $\theta_{2}$, where $\theta_{1}$ and $\theta_{2}$ correspond to a rotation around the $z^{\prime}$-axis, and $\beta$ a rotation around the $y^{\prime}$-axis. The transformation of a point $\boldsymbol{v}^{\prime}$ in the $X^{\prime} Y^{\prime} Z^{\prime}$ coordinate system into the corresponding point $v$ in the $X Y Z$ coordinate system is obtained by means of the orthogonal rotation matrices $\mathbf{M}_{\theta_{1}}$, $\mathbf{M}_{\beta}$ and $\mathbf{M}_{\theta_{2}}$, i.e.

$$
\boldsymbol{v}=\mathbf{M}_{\theta_{1}} \mathbf{M}_{\beta} \mathbf{M}_{\theta_{2}} \boldsymbol{v}^{\prime}+\boldsymbol{v}_{U} \triangleq \boldsymbol{F} \boldsymbol{v}^{\prime}+\boldsymbol{v}_{U}
$$

where $\boldsymbol{v}=(x, y, z)^{T}, \boldsymbol{v}^{\prime}=\left(x^{\prime}, y^{\prime}, z^{\prime}\right)^{T}$ and

$$
\begin{aligned}
\mathbf{M}_{\theta_{n}} & =\left(\begin{array}{ccc}
\cos \theta_{n} & -\sin \theta_{n} & 0 \\
\sin \theta_{n} & \cos \theta_{n} & 0 \\
0 & 0 & 1
\end{array}\right), n=1,2 \\
\mathbf{M}_{\beta} & =\left(\begin{array}{ccc}
\cos \beta & 0 & \sin \beta \\
0 & 1 & 0 \\
-\sin \beta & 0 & \cos \beta
\end{array}\right)
\end{aligned}
$$

The coordinate system $X^{\prime} Y^{\prime} Z^{\prime}$ is convenient to describe the receiver layout and to determine the RSS values in the PDs. Therefore, unless specified otherwise, all receiver-related expressions are given in the $X^{\prime} Y^{\prime} Z^{\prime}$ coordinate system. As mentioned earlier, the receiver consists of two parallel planes. The plane of the apertures is an opaque screen containing circular holes with radius $R_{A}$, and the PDs are circular with radius $R_{D}$. We assume that the radius $R_{A}$ is large compared to the wavelength of the light, implying the incident light will introduce a circular light spot with radius $R_{A}$ on the plane of the PDs, and we assume that the only light that reaches $\mathrm{PD} j$ is the light coming through aperture $j$. In the following, we set $R_{A}=R_{D}=h_{A}$. The centre of aperture $j$, which has position $\boldsymbol{v}_{A P, j}^{\prime}=\left(x_{A P, j}^{\prime}, y_{A P, j}^{\prime}, z_{A P, j}^{\prime}\right)^{T}$, where $z_{A P, j}^{\prime}=0$, is relatively displaced compared to the centre of PD $j$, which has position $\boldsymbol{v}_{P D, j}^{\prime}=\left(x_{P D, j}^{\prime}, y_{P D, j}^{\prime}, z_{P D, j}^{\prime}\right)^{T}=\boldsymbol{v}_{A P, j}^{\prime}+\Delta \boldsymbol{v}_{A P, j}^{\prime}$, with $\Delta v_{A P, j}^{\prime}=\left(d_{A P, j} \cos \alpha_{A P, j}^{\prime} ; d_{A P, j} \sin \alpha_{A P, j}^{\prime} ;-h_{A}\right)$. The positions of the PDs with respect to their apertures are selected to obtain a receiver with angular diversity, i.e. that can detect the direction from which light is coming.

In order to distinguish the directions in which the receiver sees the different LEDs, the receiver must be able to separate the contributions from the LEDs. We define the optical signal transmitted by LED $i$ as $s_{i}(t)$. At RE $j$, the PD converts the incoming optical signal to an electrical signal $r_{i}(t)$. To extract the contributions from the different LEDs, the receiver correlates $r_{j}(t)$ with a set of $K$ reference signals $\psi_{l}(t)$ over the interval $[0, T]^{1}$. Defining $r_{l}[j]=\int_{0}^{T} r_{j}(t) \psi_{l}(t) d t$, the $M K \times 1$ vector of observations $\boldsymbol{r}=\left(\boldsymbol{r}_{1}^{T} \ldots \boldsymbol{r}_{L}^{T}\right)^{T}$, with $\boldsymbol{r}_{l}=\left(r_{l}[1] \ldots r_{l}[M]\right)^{T}$ yields

$$
\boldsymbol{r}=R_{p} \tilde{\mathbf{H}} \boldsymbol{\mu}+\tilde{\mathbf{N}}
$$

In (3), $R_{p}$ is the responsitivity of the $\mathrm{PD}$ and $\tilde{\mathbf{H}}$ is a $M K \times K^{2}$ matrix:

$$
\tilde{\mathbf{H}}=\left(\begin{array}{cccc}
\mathbf{H} & 0 & \ldots & 0 \\
0 & \mathbf{H} & \ldots & 0 \\
\vdots & \vdots & & \vdots \\
0 & 0 & \ldots & \mathbf{H}
\end{array}\right)
$$

with $(\mathbf{H})_{j, i}=h_{c}^{(j, i)}, j=1, \ldots, M, i=1, \ldots, K$ the $M \times K$ matrix of the channel gains. Further, the $K^{2} \times 1$ vector $\boldsymbol{\mu}$ contains the contributions from the transmitted optical signals $s_{i}(t), i=$ $1, \ldots, K$, with $\boldsymbol{\mu}=\left(\boldsymbol{\mu}_{1}^{T} \ldots \boldsymbol{\mu}_{L}^{T}\right)^{T}$, where $\boldsymbol{\mu}_{l}=\left(\boldsymbol{\mu}_{l}[1] \ldots \boldsymbol{\mu}_{l}[K]\right)^{T}$ and $\boldsymbol{\mu}_{l}[i]=\int_{0}^{T} s_{i}(t) \psi_{l}(t) d t$. The contributions from the noise are contained in the $M K \times 1$ vector $\tilde{N}=\left(\boldsymbol{N}_{1}^{T} \ldots \boldsymbol{N}_{K}^{T}\right)^{T}$, with $N_{l}=\left(N_{l}[1] \ldots N_{l}[M]\right)^{T}$. Assuming the bandwidth of the signal $s_{i}(t)$ is below $10 \mathrm{MHz}$, the noise is dominated by the shot noise [19]. We assume the shot noise is modelled as a zero-mean Gaussian random variable with covariance matrix $N_{0} / 2 \cdot \tilde{\mathbf{R}}$, where $\tilde{\mathbf{R}}=\mathbf{R}_{\psi} \otimes \mathbf{I}_{M}$, with $\otimes$ denoting the Kronecker product. The elements of the $L \times L$ matrix $\mathbf{R}_{\psi}$ equal $\left(\mathbf{R}_{\psi}\right)_{l, l^{\prime}}=$ $\int_{0}^{T} \psi_{l}(t) \psi_{l^{\prime}}(t) d t$ and $\mathbf{I}_{M}$ is the $M \times M$ identity matrix . Further, the noise level $N_{0}$ is defined as $N_{0}=2 q R_{p} p_{n} A_{D} \Delta \lambda$ [19], where $q$ is the charge of an electron, $p_{n}$ is the background spectral irradiance, $A_{D}$ is the area of the PD and $\Delta \lambda$ is the bandwidth of the optical filter in front of the PD. In order to determine

${ }^{1}$ The reference signals $\psi_{l}(t)$ are related to the transmitted signals $s_{i}(t)$. Details about the signals $s_{i}(t)$ and $\psi_{l}(t)$ will be given in Section II.c. 


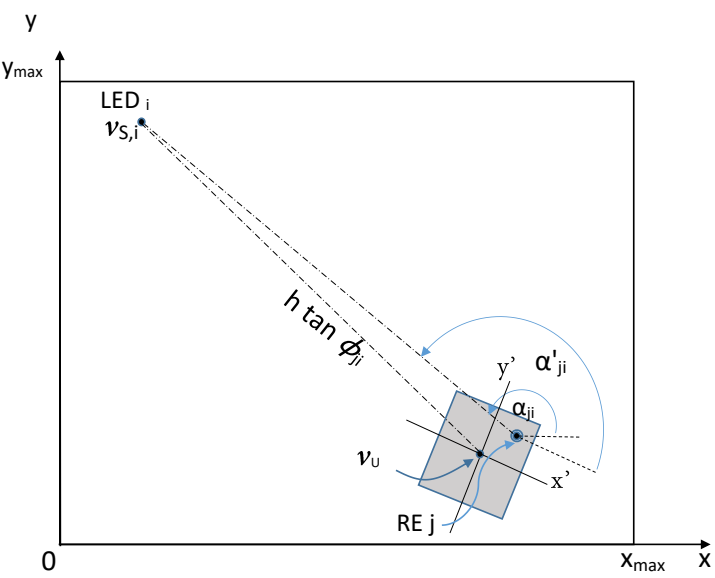

(a) TOP VIEW

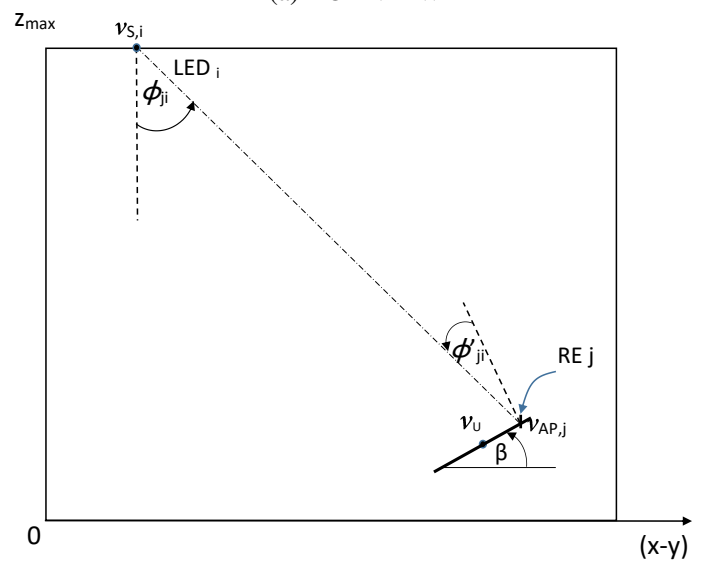

(b) SIDE VIEW

Fig. 2: Views of the room: a) TOP VIEW shows $\alpha_{j, i}, \alpha_{j, i}^{\prime}$ and the $x y$-plane where the receiver is rotated around the $z^{\prime}$-axis and b) SIDE VIEW shows the angles $\phi_{j, i}, \phi_{j, i}^{\prime}$, the height of the area and the tilt angle $\beta$. Both display the position of LED $i$, the center of the receiver $\boldsymbol{v}_{U}$ and RE $j$.

the channel gain $h_{c}^{(j, i)}$, we define the incident angles $\phi_{j, i}$ and $\phi_{j, i}^{\prime}$ and the rotation angles $\alpha_{j, i}$ and $\alpha_{j, i}^{\prime}$ (see Fig. 2) :

$$
\begin{aligned}
& x_{S, i}=x_{A P, j}+\left(z_{S, i}-z_{A P, j}\right) \tan \phi_{j, i} \cos \alpha_{j, i} \\
& y_{S, i}=y_{A P, j}+\left(z_{S, i}-z_{A P, j}\right) \tan \phi_{j, i} \sin \alpha_{j, i}
\end{aligned}
$$

and

$$
\begin{aligned}
& x_{S, i}^{\prime}=x_{A P, j}^{\prime}+\left(z_{S, i}^{\prime}-z_{A P, j}^{\prime}\right) \tan \phi_{j, i}^{\prime} \cos \alpha_{j, i}^{\prime} \\
& y_{S, i}^{\prime}=y_{A P, j}^{\prime}+\left(z_{S, i}^{\prime}-z_{A P, j}^{\prime}\right) \tan \phi_{j, i}^{\prime} \sin \alpha_{j, i}^{\prime}
\end{aligned},
$$

where $\boldsymbol{v}_{A P, j}=\left(x_{A P, j}, y_{A P, j}, z_{A P, j}\right)^{T}=\boldsymbol{F} \boldsymbol{v}_{A P, j}^{\prime}+\boldsymbol{v}_{U}$ are the coordinates of $\mathrm{AP} j$ in the $X Y Z$ coordinate system and $\boldsymbol{v}_{S, i}^{\prime}=$ $\left(x_{S, i}^{\prime}, y_{S, i}^{\prime}, z_{S, i}^{\prime}\right)^{T}=\boldsymbol{F}^{T}\left(\boldsymbol{v}_{S, i}-\boldsymbol{v}_{U}\right)$ are the coordinates of LED $i$ in the $X^{\prime} Y^{\prime} Z^{\prime}$ coordinate system. Following [19], the channel gain $h_{c}^{(j, i)}$ can be written as:

$$
h_{c}^{(j, i)}=\frac{m_{i}+1}{2 \pi\left(z_{S, i}-z_{A P, j}\right)^{2}} A_{0}^{(j, i)} \cos ^{m+2} \phi_{j, i} \cos \phi_{j, i}^{\prime}
$$

where $A_{0}^{(j, i)}$ corresponds to the overlap area between PD $j$ and the light spot originating from LED $i$ :

$$
A_{0}^{(j, i)}= \begin{cases}2 R_{D}^{2} \arccos \left(\frac{d_{j, i}}{2 R_{D}}\right) & 0 \leq d_{j, i} \leq 2 R_{D} \\ -\frac{d_{j, i}}{2} \sqrt{4 R_{D}^{2}-d_{j, i}^{2}} & \\ 0 & d_{j, i}>2 R_{D}\end{cases}
$$

This overlap area is a function of the distance $d_{j, i}$ between the centre of the light spot and the centre of PD $j$ (see Fig. $1 b$ ). Defining the position of the light spot (in the $X^{\prime} Y^{\prime} Z^{\prime}$ coordinate system) originating from LED $i$ and aperture $j$ as $\boldsymbol{v}_{p}^{\prime(j, i)}=\left(d_{p}^{(j, i)} \cos \alpha_{p}^{(j, i)}, d_{p}^{(j, i)} \sin \alpha_{p}^{(j, i)},-h_{A}\right)$, with $d_{p}^{(j, i)}=$ $h_{A} \tan \phi_{j, i}^{\prime}$ and $\alpha_{p}^{(j, i)}=\pi+\alpha_{j, i}^{\prime}$, the distance $d_{j, i}$ can be written as

$$
d_{j, i}=\left\|\boldsymbol{v}_{p}^{\prime(j, i)}-\boldsymbol{v}_{P D}^{\prime(j, i)}\right\|
$$

where $\|\cdot\|$ is the Euclidean norm.

\section{B. Cramer-Rao Bound}

One of the main indicators of the performance of a positioning system is the mean-squared error (MSE) on the estimate $\hat{\boldsymbol{v}}_{U}=\left(\hat{x}_{U}, \hat{y}_{U}, \hat{z}_{U}\right)$ of the position $\boldsymbol{v}_{U}=\left(x_{U}, y_{U}, z_{U}\right)$ of the receiver

$$
M S E=E\left[\left(x_{U}-\hat{x}_{U}\right)^{2}+\left(y_{U}-\hat{y}_{U}\right)^{2}+\left(z_{U}-\hat{z}_{U}\right)^{2}\right]
$$

As in most cases, the MSE performance can only be obtained through simulations, and the MSE depends on the considered estimation algorithm, it is common to compare the MSE with a theoretical benchmark. Such a theoretical benchmark allows to evaluate the performance of estimators and to easily evaluate the effect of the system parameters on the performance. A widespread theoretical benchmark is the Cramer-Rao lower bound (CRB) [20], which in the considered case is given by

$$
M S E \geq \operatorname{trace}\left(\boldsymbol{F}_{U}^{-1}\right)
$$

where $\operatorname{trace}(\cdot)$ is the trace operator and $\boldsymbol{F}_{U}$ is the Fisher information matrix (FIM). The FIM is a measure for the amount of information about $\boldsymbol{v}_{U}$ that can be extracted from the observed vector $\boldsymbol{r}$ (3) of RSS values, and is given as:

$$
\boldsymbol{F}_{U}=E\left[\left(\boldsymbol{\nabla}_{\boldsymbol{v}_{U}} \ln p\left(\boldsymbol{r} \mid \boldsymbol{v}_{\boldsymbol{U}}\right)\right)\left(\boldsymbol{\nabla}_{\boldsymbol{v}_{U}} \ln p\left(\boldsymbol{r} \mid \boldsymbol{v}_{\boldsymbol{U}}\right)\right)^{T}\right] .
$$

Similarly as in [2], where the CRB was derived for the two-dimensional (2D) case and for the receiver parallel to the ceiling, we can derive the FIM for the three-dimensional (3D) case and other receiver orientations:

$$
\mathbf{F}_{U}=\frac{2 R_{p}^{2}}{N_{0}}\left[\begin{array}{lll}
Z^{x_{U} x_{U}} & Z^{x_{U} y_{U}} & Z^{x_{U} z_{U}} \\
Z^{y_{U} x_{U}} & Z^{y_{U} y_{U}} & Z^{y_{U} z_{U}} \\
Z^{z_{U} x_{U}} & Z^{z_{U} y_{U}} & Z^{z_{U} z_{U}}
\end{array}\right]
$$

To obtain (13), we inserted the distribution $p\left(\boldsymbol{r} \mid \boldsymbol{v}_{U}\right)$, i.e. $\boldsymbol{r} \mid \boldsymbol{v}_{U} \sim$ $N\left(R_{p} \tilde{\mathbf{H}} \boldsymbol{\mu}, \frac{N_{0}}{2} \tilde{\mathbf{R}}\right)$, and define

$$
Z^{a b}=\operatorname{trace}\left(\mathbf{R}_{\psi}^{-1} \mathbf{W}^{a b}\right),
$$


with $a, b \in\left\{x_{U}, y_{U}, z_{U}\right\},\left(\mathbf{W}^{a b}\right)_{l, l^{\prime}}=\boldsymbol{\mu}_{l}^{T} \mathbf{X}^{a b} \boldsymbol{\mu}_{l^{\prime}}$ and

$$
\begin{aligned}
\left(\mathbf{X}^{a b}\right)_{i, i^{\prime}} & =\left[\left(\frac{\partial}{\partial a} \mathbf{H}\right)^{T}\left(\frac{\partial}{\partial b} \mathbf{H}\right)\right]_{i, i^{\prime}} \\
& =\sum_{j=1}^{M} \frac{\partial}{\partial a} h_{c}^{(j, i)} \frac{\partial}{\partial b} h_{c}^{\left(j, i^{\prime}\right)} .
\end{aligned}
$$

The derivation of $\frac{\partial}{\partial a} h_{c}^{(j, i)}$ is tedious but straightforward and therefore is omitted in this paper.

\section{Transmitted Optical Signal}

The transmitted optical signals must be positive and realvalued. Further, as mentioned in Section II.a, the signals must be selected properly to be able to separate them at the receiver. A signal format that is often considered for visible light communication is optical orthogonal frequency division multiplexing (O-OFDM) [21], [22], [23]. Adopting this signal format to the VLP system, each LED is assigned a DC-biased windowed sinusoid waveform with duration $T$ :

$$
s_{i}(t)=A_{i} w(t)\left(1+\cos \left(2 \pi f_{c, i} t\right)\right),
$$

where the frequencies $f_{c, i}, i=1, \ldots, K$ are selected so that $f_{c, i} T$ and $\left(f_{c, i}-f_{c, i^{\prime}}\right) T$ are integer, i.e. the sinusoids are orthogonal over the interval $[0, T]$, and $w(t)$ is a window function, e.g. a raised-cosine function:

$$
w(t)=1+\cos \left(\frac{2 \pi}{T}\left(t-\frac{T}{2}\right)\right) .
$$

We assume the bandwidth of the window function is narrow compared to the frequencies $f_{c, i}$ of the sinusoids. Defining the optical power $P_{i}$ transmitted by LED $i$ as:

$$
P_{i}=\frac{1}{T} \int_{0}^{T} s_{i}(t) d t,
$$

it can be verified that $P_{i}=A_{i}$, i.e. the scaling factor in (16) is the transmitted optical power.

At the receiver, the PDs detect the sum of the contributions from the different LEDs. Because all LEDs contribute to the DC component, employing the DC component for positioning purposes would result in interference between the optical signals. To avoid performance reduction due to this interference, we remove at the receiver the DC component from the signal by correlating the received signal with the reference signals $\psi_{l}(t)=A_{l} w(t) \cos \left(2 \pi f_{c, l} t\right)$. In that case, $\boldsymbol{\mu}_{l}$ and $\boldsymbol{R}_{\psi}$ reduce to:

$$
\begin{aligned}
\boldsymbol{\mu}_{l}[i] & =\frac{3 T}{4} A_{i} \boldsymbol{\delta}_{i, l} \\
\mathbf{R}_{\psi} & =\frac{3 T}{4} \mathbf{I}_{K}
\end{aligned}
$$

where $\mathbf{I}_{K}$ is the $K \times K$ identity matrix.

\section{NUMERICAL RESULTS}

In this section, we will numerically evaluate the CRB. We consider a receiver that contains $M=8 \mathrm{REs}$, with positions of the centres of the apertures $v_{A P, j}^{\prime}=\left(x_{A P, j}^{\prime}, y_{A P, j}^{\prime}, z_{A P, j}^{\prime}\right)$, where

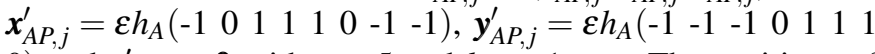
$0)$ and $z_{A P, j}^{\prime}=0$ with $\varepsilon=5$ and $h_{A}=1 \mathrm{~mm}$. The positions of the centres of the PDs, relative to the centres of the apertures, i.e. $\Delta v_{A P, j}^{\prime}=\left(d_{A P, j} \cos \alpha_{A P, j}^{\prime}, d_{A P, j} \sin \alpha_{A P, j}^{\prime},-h_{A}\right)$ are selected so that $d_{A P, j}=0.5 R_{D}$ and $\alpha_{A P, j}^{\prime}=j \cdot \frac{\pi}{4}$. Next, for the transmitters, we consider $K=P^{2}$ LEDs, with Lambertian order $m_{i}=1$, that are placed in a rectangular grid with spacing $\Delta x_{L E D}$ and $\Delta y_{L E D}$ in the $X$ and $Y$ directions, i.e.

$$
\begin{aligned}
& x_{S}(i)=\frac{x_{\max }}{2}-\frac{\Delta x_{L E D}}{2}\left((P-1)-2(i-1)_{P}\right) \\
& y_{S}(i)=\frac{y_{\max }}{2}-\frac{\Delta y_{L E D}}{2}\left((P-1)-2\left\lfloor\frac{i-1}{P}\right\rfloor\right),
\end{aligned}
$$

for $i=1, \ldots, K$, with $(x)_{P}$ the modulo- $P$ reduction of $x$ and $\lfloor x\rfloor$ the largest integer not exceeding $x$. Further, $x_{\max }$ and $y_{\max }$ denote the length and width of the area in which the CRB is evaluated. In the following, we assume $x_{\max }=y_{\max }=10$ $\mathrm{m}$. We suppose all LEDs have the same transmit power, i.e. $P_{i}=A_{i}=A=1 \mathrm{~W}$. In that case, $\mathbf{A} \circ \mathbf{A}=A^{2} \mathbf{I}_{K}$. Furthermore, to determine the shot noise spectral density level, we assume a background spectral irradiance $p_{n}=5.8 \times 10^{-6} \mathrm{~W} / \mathrm{cm}^{2} \cdot \mathrm{nm}$ [19], the PD responsitivity $R_{p}=0.4 \mathrm{~mA} / \mathrm{mW}$ [24] and an optical filter passing only visible light frequencies in the range 380 to $740 \mathrm{~nm}$, resulting in an optical bandwidth of $\lambda=360$ $\mathrm{nm}$. This results in a noise spectral density $N_{0}=8.4 \times 10^{-24}$ $\mathrm{A}^{2} / \mathrm{Hz}$. Finally, the time window length $T$ equals $T=1 \mathrm{~ms}$.

First, we evaluated the effect of the tilt $\beta$ on the CRB. We assume $K=4$ and a uniform distribution of the LEDs over the area, i.e. $\Delta x_{L E D}=\Delta y_{L E D}=\frac{x_{\max }}{P}$. In Fig. 3, we show the root of the $\mathrm{CRB}$, i.e. $\mathrm{rCRB}$, as function of the position of the receiver, for different tilt angles $\beta$. As can be observed, when $\beta$ increases, some positions will experience a performance degradation, while other positions will have better performance compared to the case where the receiver is parallel to the ceiling, i.e. when $\beta=0$. This can be explained as follows. In our example, the receiver is tilted to the right, i.e. the $z^{\prime}$-axis points to the right hand side of the area shown in Fig. 3. Because of this tilt, receiver positions in the left part of the area will receive more light, as - on the average the incident angles $\phi_{j, i}^{\prime}$ reduce. As a result, these positions will show improved performance compared to the case $\beta=0$, although the gain is modest to small. On the other hand, in the right part of the area, the receiver is pointing away from the LEDs. As on the average, the incident angles $\phi_{j, i}^{\prime}$ are increased, the REs will receive less light, resulting in a performance degradation. We can conclude that, as intuitively could be expected, the performance improves when pointing towards the LEDs, while it degrades when the receiver points away from the LEDs.

The strong degradation in Fig. 3 can be attributed to the small number of LED used. As only 4 LEDs are present, the relative horizontal distances between the LEDs and the receiver are relatively large, indicating the incident angles $\phi_{j, i}^{\prime}$ will also be relatively large. By tilting the receiver, the resulting angles $\phi_{j, i}^{\prime}$ may quickly become too large, so that the receiver will no longer capture the light from one or more LEDs, even if the tilt angle is moderate. We predict that the degradation caused by the loss of a LED, because it is no longer in the field-of-view (FOV) of the receiver, will reduce when the number of LEDs increases. Due to the closer spacing between the LEDs, the incident angles $\phi_{j, i}^{\prime}$ between the receiver and the nearest LEDs will become relatively smaller when $K$ increases. Hence, we can tolerate larger tilts before the LEDs disappear out of the FOV of the receiver, and only when the number of LEDs that are within the FOV becomes 


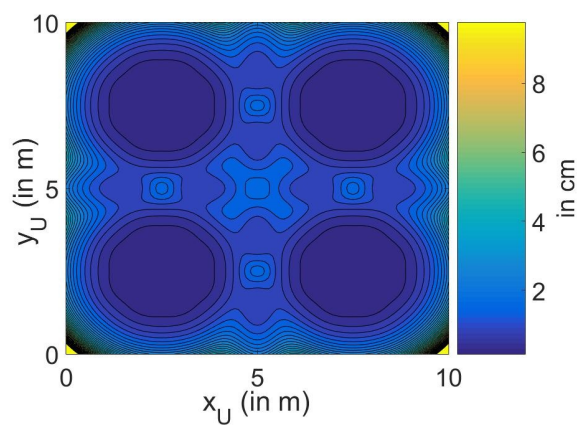

(a)

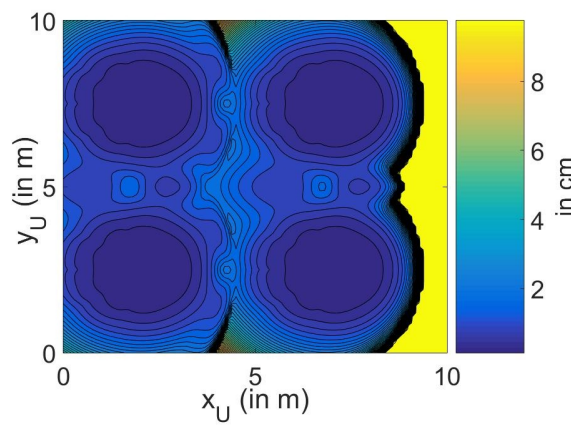

(b)

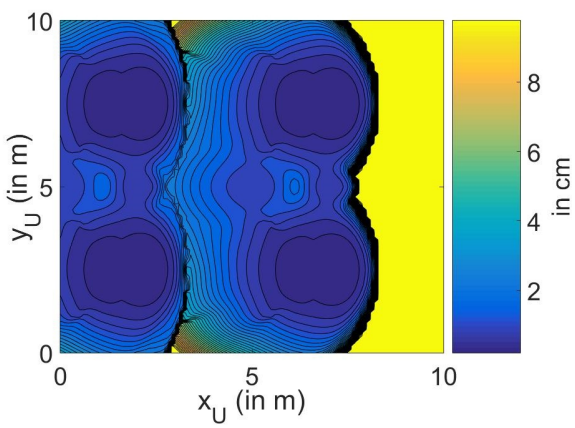

(c)

Fig. 3: Square root of the CRB as function of the position $\left(x_{U}, y_{U},-2 \mathrm{~m}\right)$ of the receiver in the area, for $A_{i}=1 \mathrm{~mW}, K=4$ and tilt angle: (a) $\beta=0$, (b) $\beta=\pi / 8$ and (c) $\beta=\pi / 4$

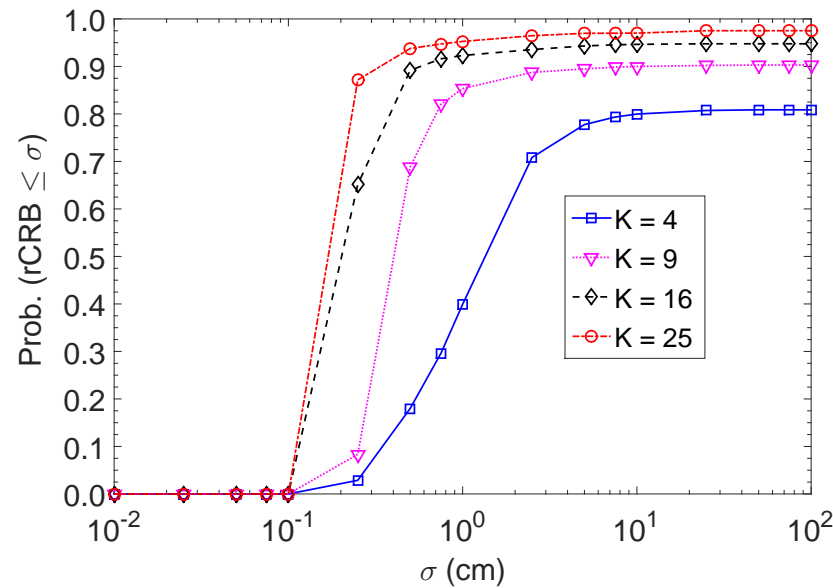

Fig. 4: Probability of the $\mathrm{rCRB} \leq \sigma$ as function of the number of LEDs with $z_{U}=-2 \mathrm{~m}, \theta_{1}=\theta_{2}=0$ and $\beta=\pi / 4$.

too small, a strong degradation or the inability to determine the position will occur. When the number of LEDs increases, the probability that this will happen reduces. This can be observed in Fig. 4 , where a tilt angle $\beta=\pi / 4$ and $z_{U}=-2 \mathrm{~m}$ are considered. While for $K=4$, the position cannot be determined accurately, i.e. with rCRB $<10 \mathrm{~cm}$, in $20 \%$ of the positions, this percentage reduces when $K$ increases, i.e. for $K=25$, only in $3 \%$ of the positions, $\mathrm{rCRB}>10 \mathrm{~cm}$. In our simulations, we noticed that when the tilt angle $\beta>\pi / 4$, some of the receiver positions could not capture sufficient light to determine the receiver position, unless the number of LEDs was very high. Hence, in the reminder of the paper, we restrict our attention to tilt angles in the interval $\beta=[0, \pi / 4]$.

Next, we evaluate the average of the rCRB, i.e. the average over all receiver positions in the considered area, as function of the Euler angles $\theta_{1}, \theta_{2}$ and $\beta$. The results, which are shown in Fig. 5, illustrate that the rotations $\theta_{1}$ and $\theta_{2}$ have no noticeable effect on the average rCRB. This is due to the symmetry of the receiver. On the other hand, the previous results showed that the tilt angle has a larger influence on the performance. As can be observed in Fig. 5, the average rCRB is the smallest for $\beta=0$, and increases when $\beta$ increases. By tilting the receiver, although some receiver positions will benefit from this tilt and have slightly better performance, other positions will experience a large performance degradation, so that the average rCRB increases compared to $\beta=0$. The average rCRB will increase when $\beta$ increases, as when the tilt angle grows, the amount of positions that have degraded performance rises. As can be observed in Fig. 5a, when $K=16$, the average rCRB increases from $0.15 \mathrm{~cm}$ for $\beta=0$ to almost $0.3 \mathrm{~cm}$ for $\beta=\pi / 6$. However, although the average rCRB almost doubles, we still obtain centimetre accuracy for a wide range of tilt angles.

In the previous results, we assumed that the receiver was located $2 \mathrm{~m}$ below the ceiling, i.e. $z_{U}=-2 \mathrm{~m}$. Now, we will look at the influence of the vertical distance between the receiver and the LEDs on the sensitivity of the rCRB to the tilt angle. In Fig. 6, we show the probability of the rCRB being smaller than $\sigma$, for different angles $\beta$ and distances $z_{U}$. As can be observed, when the vertical distance becomes smaller, the amount of receiver positions where the position cannot be determined grows. This can be explained as when $\left|z_{U}\right|$ decreases, the incident angles $\phi_{j, i}^{\prime}$ and $\phi_{j, i}$ become relatively larger, so that a LED will disappear out of the FOV of the receiver at a smaller tilt angle.

Finally, we investigate how the sensitivity to a tilt is influenced by the spacing of the LEDs. To this end, we consider $K=16$ LEDs that are placed in a square grid, where we vary the distance $\Delta x_{L E D}=\Delta y_{L E D}$ (20) between the LEDs. The results are shown in Fig. 7. A first observation is that, when the spacing decreases, the probability that, for some of the positions in the area, the receiver is unable to determine its position increases, especially if the tilt angle increases. Because the LEDs are closer to the centre of the area, the incident angles $\phi_{j, i}^{\prime}$ for a receiver that is located near the edges of the area increases. Hence, we can only tolerate a smaller tilt before too many LEDs disappear out of the FOV of the receiver. A second observation is that the differences between the curves are very small. This indicates that, if the receiver is able to locate itself because sufficient LEDs are within its FOV, the tilt has a negligible effect on the performance. Also, it implies that the degradation mainly occurs because insufficient LEDs are within the FOV of the receiver. Finally, we observe that the lowest average $\mathrm{rCRB}$ is obtained when $\Delta x_{L E D}=250$ 


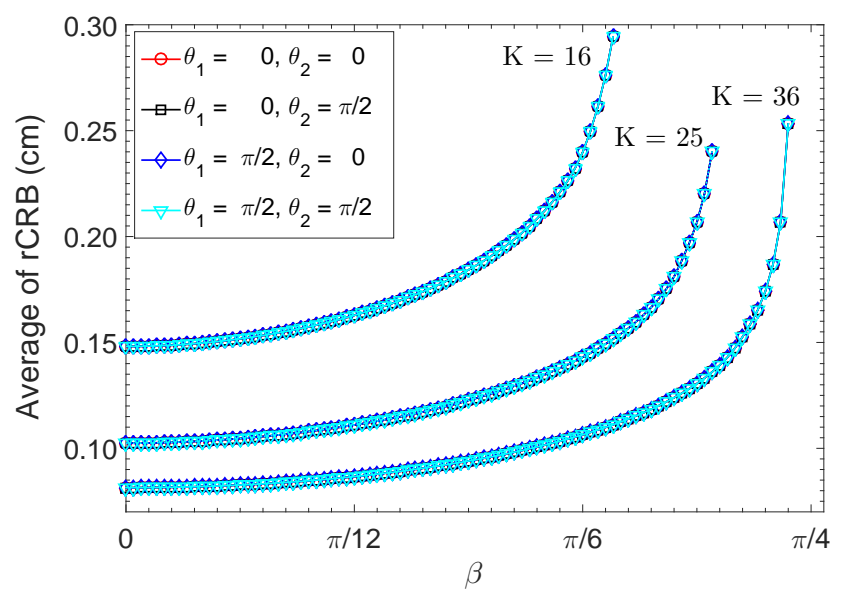

(a)

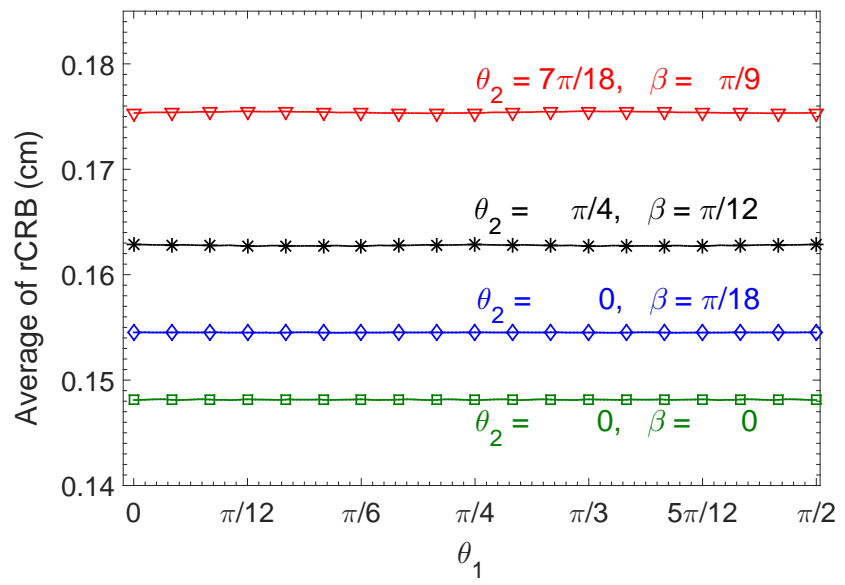

(b)

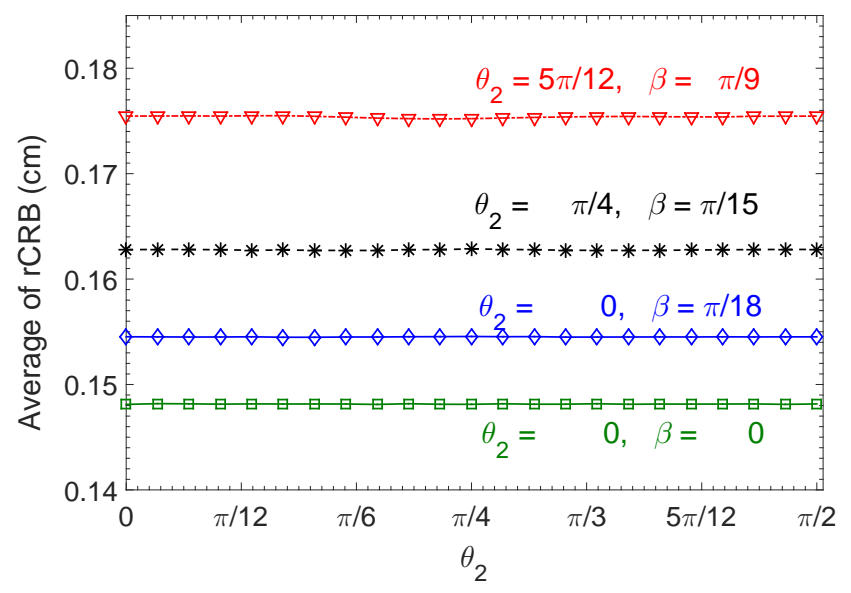

(c)

Fig. 5: The average of rCRB for $z_{U}=-2 \mathrm{~m}, \Delta x_{L E D}=\Delta y_{L E D}=x_{\max } / P$ as a function of the Euler angles: (a) tilt $\beta$ for $K=16,25,36$, (b) rotation $\theta_{1}$ for $K=16$ and (c) rotation $\theta_{2}$ for $K=16$.

$\mathrm{cm}$. This corresponds to uniformly distributing the 16 LEDs over the $10 \mathrm{~m} \times 10 \mathrm{~m}$ area.

\section{CONCLusions}

In this paper, we investigate the effect of the receiver orientation on the CRB for the mean squared positioning error, for an aperture-based receiver where the position is directly

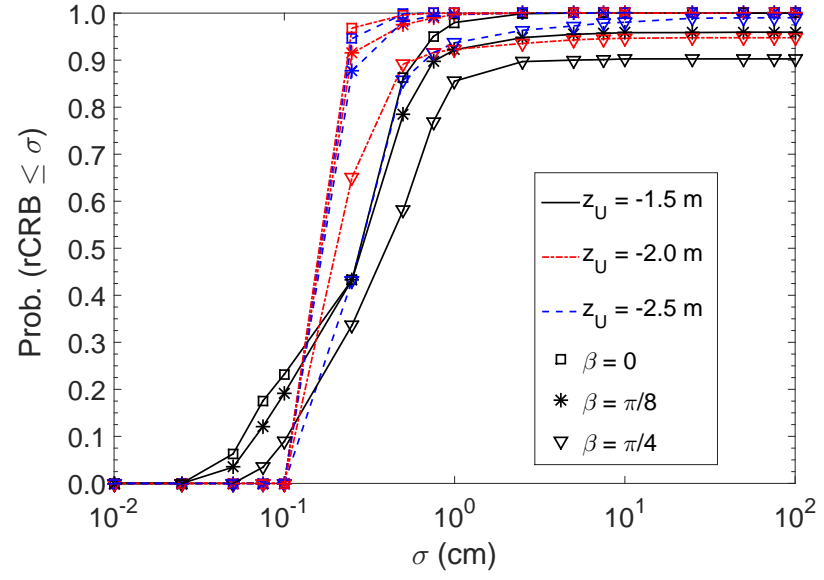

Fig. 6: Probability of the $r C R B \leq \sigma$ as function of a level of accuracy required, $\sigma$, for $K=16$ LEDs, $\Delta x_{L E D}=\Delta y_{L E D}=x_{\max } / P$ and $\theta_{1}=\theta_{2}=0$

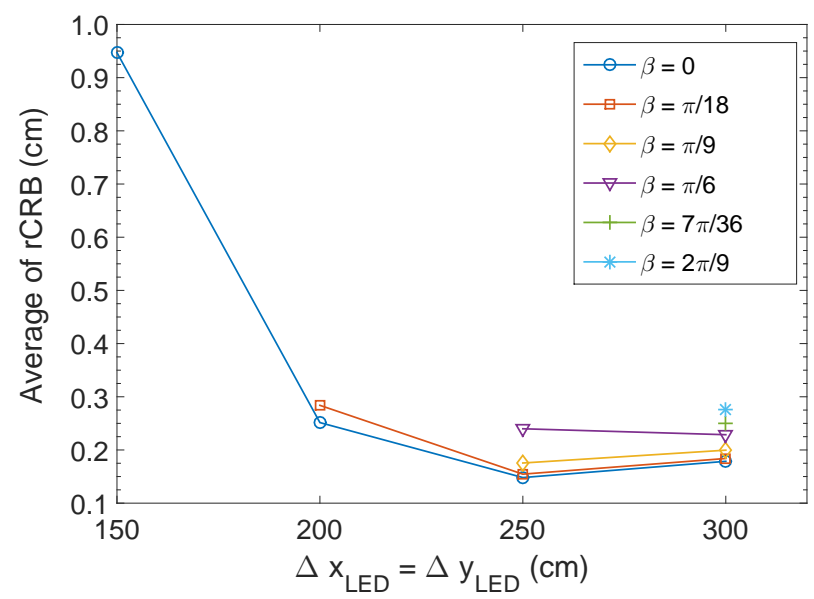

Fig. 7: Values of average of rCRB for different distance of separation between LEDs for $K=16, z_{U}=-2 \mathrm{~m}$ and $\theta_{1}=\theta_{2}=0$.

estimated from the measured RSS values. We describe the receiver orientation by introducing three Euler angles, $\theta_{1}, \beta$ and $\theta_{2}$, where $\theta_{1}$ and $\theta_{2}$ are rotations around the normal to the plane of the PDs, and $\beta$ is the tilt of the receiver. We show that the rotations $\theta_{1}$ and $\theta_{2}$ have no effect on the performance. This is explained by the symmetry of the receiver. The tilt has a larger effect on the CRB, as it influences the orientation of the FOV of the receiver. We show that, when the receiver is tilted, the performance is degraded when too few LEDs are within the FOV of the receiver. However, as long as the receiver can detect a sufficient number of LEDs, the tilt has almost no effect on the performance. We show that centimetre accuracy can be obtained for a wide range of tilt angles.

\section{ACKNOWLEDGEMENT}

José Miguel Menéndez acknowledges the National Secretariat of Higher Education, Science, Technology and Innovation of Ecuador (SENESCYT) for their financial support.

\section{REFERENCES}

[1] T. Q Wang, C. He, and J. Armstrong. Angular diversity for indoor mimo optical wireless communications. In 2015 IEEE International Conference on Communications (ICC), pages 5066-5071. IEEE, 2015. 
[2] H. Steendam, T.Q. Wang, and J. Armstrong. Theoretical lower bound on vlc-based indoor positioning using received signal strength measurements and an aperture-based receiver. Journal of Lightwave Technology, 35(2):309 - 319, Jan 2017.

[3] Y. Wang, X. Yang, Y. Zhao, Y. Liu, and L. Cuthbert. Bluetooth positioning using rssi and triangulation methods. In 2013 IEEE 10th Consumer Communications and Networking Conference (CCNC), pages 837-842. IEEE, 2013.

[4] C. Yang and H.-R. Shao. Wifi-based indoor positioning. IEEE Communications Magazine, 53(3):150-157, 2015.

[5] G. Shi and Y. Ming. Survey of indoor positioning systems based on ultrawideband (uwb) technology. In Wireless Communications, Networking and Applications, pages 1269-1278. Springer, 2016.

[6] H. S. Liu and G. Pang. Positioning beacon system using digital camera and leds. IEEE Transactions on Vehicular Technology, 52(2):406-419, 2003.

[7] T. Nakamura and T. Takebe. Development of znse-based white light emitting diodes. OPTRONICS, 19(228):126-131, 2000.

[8] T. Komine. Visible light wireless communications and its fundamental study. PhD thesis, Keio University, 2005.

[9] C. P. Kuo, R. M. Fletcher, T. D. Owentowski, M. C. Lardizabal, and M. G. Craford. High performance algainp visible light-emitting diodes. Appl. Phys. Lett., 57(27):2937-2939, 1990.

[10] Z. Zhou, M. Kavehrad, and P. Deng. Indoor positioning algorithm using light-emitting diode visible light communications. Optical Engineering, 51(8):085009-1, 2012.

[11] S.-H. Yang, E.-M. Jeong, D.-R. Kim, H.-S. Kim, Y.-H. Son, and S.-K Han. Indoor three-dimensional location estimation based on led visible light communication. Electronics Letters, 49(1), January 2013.

[12] G. Kail, P. Maechler, N. Preyss, and A. Burg. Robust asynchronous indoor localization using led lighting. IEEE International Conference on Acoustics, Speech and Signal Processing (ICASSP2014), 2014.

[13] M. Rahaim, G. B Prince, and T. DC Little. State estimation and motion tracking for spatially diverse vlc networks. In 2012 IEEE Globecom Workshops, pages 1249-1253. IEEE, 2012.

[14] A. Arafa, X. Jin, and R. Klukas. Wireless indoor optical positioning with a differential photosensor. IEEE Photonics Technology Letters, 32(14):2480-2485, Jul. 2012.

[15] Y.U. Lee and S.-M. Lee. Random distributed angle-of-arrival parameter estimation technique for visible light positioning. in Proc. Int. Conf. on Telecom. and Sign. Proc., TSP2015, pages 467-471, 2015.

[16] T.Q. Wang, Y.A. Sekercioglu, A. Neild, and J. Armstrong. Position accuracy of time-of-arrival based ranging using visible light with application in indoor localization systems. Journal of Lightwave Technology, 31(20):3302-3308, October 2013.

[17] U. Nadeem, N.U. Hassan, M.A. Pasha, and C. Yuen. Highly accurate 3d wireless indoor positioning system using white led lights. Electronics Letters, 50(11):828-830, May 2014.

[18] D. E. Manolakis. Efficient solution and performance analysis of 3-d position estimation by trilateration. IEEE Transactions on Aerospace and Electronic Systems, 32(4):1239-1248, 1996.

[19] J. M. Kahn and J. R. Barry. Wireless infrared communications. Proceedings of the IEEE, 85(2):265-298, 1997.

[20] H. L. Van Trees. Detection, Estimation, and Modulation Theory, Part III. John Wiley \& Sons, 2001.

[21] J. B. Carruthers and J. M. Kahn. Multiple-subcarrier modulation for nondirected wireless infrared communication. IEEE Journal on Selected Areas in Communications, 14(3):538-546, 1996.

[22] O. González, R. Perez-Jimenez, S Rodriguez, J. Rabadán, and A. Ayala. Ofdm over indoor wireless optical channel. IEE ProceedingsOptoelectronics, 152(4):199-204, 2005.

[23] J. Armstrong. Ofdm for optical communications. Journal of lightwave technology, 27(3):189-204, 2009.

[24] L. Zeng, D.C. O’Brien, H.L. Minh, G.E. Faulkner, K. Lee, D. Jung, Y. Oh, and E.T. Wong. High data rate multiple input multiple output (mimo) optical communications using white led lighting. IEEE Journal on Selected Areas in Communications, 27(9):1654-1662, December 2009. 